\title{
Crystallization and Preliminary X-Ray Diffraction Data of Two Different Human Low-Density Lipoprotein (LDL) Subfractions
}

\author{
Stephan Ritter, ${ }^{1}$ Ingrid Frey, ${ }^{1}$ Kay Diederichs, ${ }^{2}$ Dominik Grathwohl, ${ }^{1}$ J oseph Keul, ${ }^{1}$ \\ and Manfred W. Baumstark ${ }^{1 *}$ \\ ${ }^{1}$ MedizinischeUniversitätsklinik Freiburg, D-79106 Freiburg, Germany \\ 2Fakultät für Biologie, Universität Konstanz, D-78434 Konstanz, Germany
}

\begin{abstract}
Human LDL subfractions LDL-2 $(\mathrm{d}=1.031-1.034 \mathrm{~g} / \mathrm{ml})$ and LDL-5 $(d=1.040-1.044 \mathrm{~g} / \mathrm{ml})$ were crystallized in two different crystal forms by using polyethylene glycol as a precipitant. Both fractions were from one donor. Crystals of LDL -5 were yellow, hexagonal, and showed no dichroism. Crystals of LDL-2 were of the same color, had a rodlike shape with notches at both ends, and were highly dichroitic. LDL-2 crystals diffracted to a resolution of $29 \AA$ by using synchrotron radiation. Indexing in $\mathrm{P} 1$ resulted in preliminary parameters for the reduced cell of $a=171 \AA$, $b=438 \AA, c=519 \AA, \alpha=102^{\circ}, \beta=99^{\circ}, \gamma=91$. These dimensions are consistent with the size of LDL particles. Using Fourier transform infrared spectroscopy (FTIR) and agarose gel electrophoresis, we could further confirm that the crystals consist of LDL. The FTIR spectrum showed bands characteristic for lipids and protein. Dissolved crystals exhibited a mobility similar to native LDL in agarose gels and could be stained with anti-human apolipoprotein B (apoB). Proteins 28:293-297, 1997.

๑) 1997 Wiley-Liss, Inc.
\end{abstract}

Key words: crystallization; X-ray diffraction; human low-density lipoprotein subfractions

\section{INTRODUCTION}

LDL particles play a major role in the development of coronary heart disease. A certain subclass of LDL particles, small dense LDL, has been identified as a major determinant of the severity and progression of atherosclerosis in humans. ${ }^{1,2}$ LDL particles consist of a single polypeptide chain (apoB) with a molecular weight of $510 \mathrm{kDa}(550 \mathrm{kDa}$ with carbohydrate) and a lipid moiety, which is made up of a core of cholesterol esters and triacylglycerol, surrounded by a monolayer of phospholipids. The total molecular weight of the particles depends on the number of associated lipid molecules and is between 2000 and $3000 \mathrm{kDa}$. The primary structure of apoB is known. 3,4 Spectroscopic methods have been used to investigate the secondary structure of apoB. Commonly accepted is a high $\beta$-sheet content of apoB, compared with other apolipoproteins that are predominantly $\alpha$-helical. ${ }^{5}$ The radially averaged structure of LDL particles has been studied by small-angle $X$-ray and neutron scattering. ${ }^{6-9}$ Three different LDL subfractions have been investigated by Baumstark et al. ${ }^{10,11}$

Few data on the three-dimensional structure of LDL, particularly apoB, are available, and no crystallization of a native lipoprotein has yet been reported. In addition to its basic relevance, the analysis of the three-dimensional structure of apoB is an important approach to explain the mechanisms involved in the development of atherosclerosis on a molecular basis. Of special interest would be to compare the structure of apoB in LDL particles from LDL subfractions of different atherogenic potential. ${ }^{1} \mathrm{~A}$ major prerequisite to obtain such information is the crystallization of native LDL particles. Here, we report crystallization conditions of two subfractions of LDL particles from human plasma.

\section{Sample Preparation \\ METHODS}

EDTA plasma from one single donor was obtained by standard procedures. Total LDL $(\mathrm{d}=1.019-1.063$ $\mathrm{g} / \mathrm{ml}$ ) was prepared by sequential flotation ${ }^{12}$ and fractionated into six density dasses by equilibrium density gradient centrifugation as described previously. ${ }^{10}$ The density ranges of the subfractions as determined by precision refractometry 12 of blank gradients were as follows: LDL-1, <1.031; LDL-2, 1.031-1.034; LDL-3, 1.034-1.037; LDL-4, 1.0371.040; LDL-5, 1.040-1.044; LDL-6, >1.044 g/ml. All centrifugation steps were performed at a temperature of $18^{\circ} \mathrm{C}$ by using partially filled $(6 \mathrm{ml})$ polycarbonate bottles in a $50 \mathrm{Ti}$ rotor. Phospholipid (PL), free cholesterol (FC), total cholesterol (TC), and triglycerides (TG) weremeasured by automated (EPOS, E ppendorf, Hamburg, Germany) enzymatic methods. Reagent kits for $P L, T C, F C$, and TG were from Boehringer (Mannheim, Germany), kits for PL from

Correspondence to: M.W. Baumstark, Medizinische Universitätsklinik Freiburg, Hugstetter Str. 55, D-79106 Freiburg, Germany.

Received 2 December 1996; Accepted 12 December 1996 
TABLE I. Lipid Molecules per LDL Particle [mol/mol LDL], Calculated Radius, and Calculated Molecular Weight (MW)*

\begin{tabular}{llllrrl}
\hline & PL & FC & CE & TG & Radius (nM) & MW (kDa) \\
\hline LDL-1 & $849 \pm 78$ & $827 \pm 74$ & $2053 \pm 158$ & $187 \pm 50$ & $10.41 \pm 0.26$ & $2988 \pm 210$ \\
LDL-2 & $772 \pm 63$ & $720 \pm 98$ & $1912 \pm 138$ & $138 \pm 32$ & $10.11 \pm 0.23$ & $2754 \pm 177$ \\
LDL-3 & $740 \pm 111$ & $695 \pm 149$ & $1855 \pm 255$ & $117 \pm 30$ & $9.98 \pm 0.40$ & $2664 \pm 316$ \\
LDL-4 & $626 \pm 58$ & $585 \pm 64$ & $1608 \pm 133$ & $84 \pm 25$ & $9.54 \pm 0.23$ & $2343 \pm 159$ \\
LDL-5 & $573 \pm 48$ & $520 \pm 50$ & $1535 \pm 101$ & $70 \pm 17$ & $9.36 \pm 0.19$ & $2217 \pm 124$ \\
LDL-6 & $520 \pm 18$ & $443 \pm 30$ & $1381 \pm 56$ & $73 \pm 11$ & $9.10 \pm 0.08$ & $2049 \pm 49$ \\
\hline
\end{tabular}

$* \mathrm{~N}=7$ determinations of the same donor. Values are given as mean \pm SD. For details of the calculation see Ref. 10.

bióMerieux (Nürtingen, Germany). Cholesterol ester (CE) was calculated as the molar difference between TC and FC. Apolipoprotein B was measured with endpoint nephelometry (Behring, Marburg, Germany). The assay was standardized as described in detail by Baumstark et al. ${ }^{10}$ In short, this corresponds to the Center of Disease Control (CDC) standard. 13

All subfractions were dialyzed against buffer $\mathrm{A}$ (20 $\mathrm{mM}$ Pipes, $150 \mathrm{mM}\left[\mathrm{NH}_{4}\right]_{2} \mathrm{SO}_{4}, 2 \mathrm{mM}$ ascorbate, 2 $\mathrm{mM}$ sodium azide, $2 \mathrm{mM}$ EDTA, $\mathrm{pH}$ 7.5) for $24 \mathrm{~h}$ and concentrated by ultrafiltration with a YM 100 membrane (Centricon, Amicon, Witten, Germany). The final protein concentration was $\sim 5-6 \mathrm{mg} / \mathrm{ml}$.

\section{Crystallization}

Crystallization conditions for LDL subfractions were established by using the sparse matrix screening method. ${ }^{14}$ Reagents were obtained from Hampton Research (Laguna Hills, CA). Screening was performed with the hanging drop vapor-diffusion technique at room temperature and $4^{\circ} \mathrm{C}$. The droplet volume was $8 \mu \mathrm{l}$, and the reservoir volume was $200 \mu$ l.

\section{Agarose Gel Electrophoresis and Immunoblotting}

Crystals of LDL-5 were collected with an X-ray capillary, washed three times with reservoir solution, and dissolved in $10 \mu \mathrm{l}$ of $150 \mathrm{mM} \mathrm{NaCl}$. The LDL solutions used for crystallization served as control lanes after dilution by a factor of $10^{3}-10^{4}$. Native gel electrophoresis was done on agarose gels (HDL plus-8 plates, Helena REP systems, Helena, Freiburg, Germany) according to the manufacturer's protocol. For immunoblotting, the LDL subfractions were transferred overnight to activated I mmobilon $P$ membranes (Millipore, Bedford, MA). The primary antibody was polyclonal anti-human apoB (I mmuno, Wien, Austria) at a dilution of 1:20. To get a high sensitivity, the bound primary antibodies were visualized by using a biotin-avidin system according to the manufacturer's instructions (Vector Laboratories, Burlingame, CA).

\section{FTIR Spectroscopy}

For FTIR spectroscopy, LDL-5 was crystallized on $\mathrm{a} \mathrm{CaF}_{2}$ window. The crystals were washed twice with distilled water and dried on the window at room temperature. Spectra of both the crystals and the subfractions from the density gradient were collected on a Bruker IFS 113v FTIR spectrophotometer (Bruker, Karlsruhe, Germany) equipped with an MCT detector. The spectrum of the crystals was measured against a $\mathrm{CaF}_{2}$ window as a reference. To improve the signal-to-noise ratio, 1024 scans were averaged for each sample.

\section{X-Ray Data Collection}

Resolution tests were performed on beamlines X11 and BW7B at the Deutsches Elektronensynchrotron (DESY, Hamburg, Germany) and at BL19 at the ESRF (European Synchrotron Radiation Facility, Grenoble, France). The data reported here were collected by using an 18-cm-diameter image plate detector (MarResearch) at BW7B, using a detector distance of $400 \mathrm{~mm}$ and a wavelength of $0.881 \AA$. Images were collected at room temperature with 5000-15,000 counts per frame at different Phi angles. For data evaluation, the programs IPDISP and $\mathrm{XDS}^{15}$ were used.

\section{RESULTS \\ Chemical Characterization of LDL Samples}

Averaged over $\mathrm{n}=7$ preparations, our donor had serum cholesterol values of $242 \pm 24 \mathrm{mg} / \mathrm{dll}$. Triglycerides were $157 \pm 39 \mathrm{mg} / \mathrm{dl}$. The apoB concentration in the six LDL (LDL-1-LDL-6) subfractions was $11.6 \pm 1.6,6.0 \pm 0.9,8.9 \pm 2.2,17.6 \pm 3.6,22.5 \pm 2.6$, and $20.1 \pm 4.7 \mathrm{mg} / \mathrm{dl}$. The lipid composition of the six subfractions is given in Table 1 .

\section{Crystallization}

Crystals of LDL- 5 were obtained at room temperature in droplets containing protein at a concentration of $3 \mathrm{mg} / \mathrm{ml}$, polyethylene glycol monomethyl ether 2000 as a precipitant at a concentration of $6 \%$ $(\mathrm{w} / \mathrm{w})$, and $75 \mathrm{mM}$ ammonium sulfate in $75 \mathrm{mM}$ acetate buffer, pH 4.6. We obtained small hexagonal, 


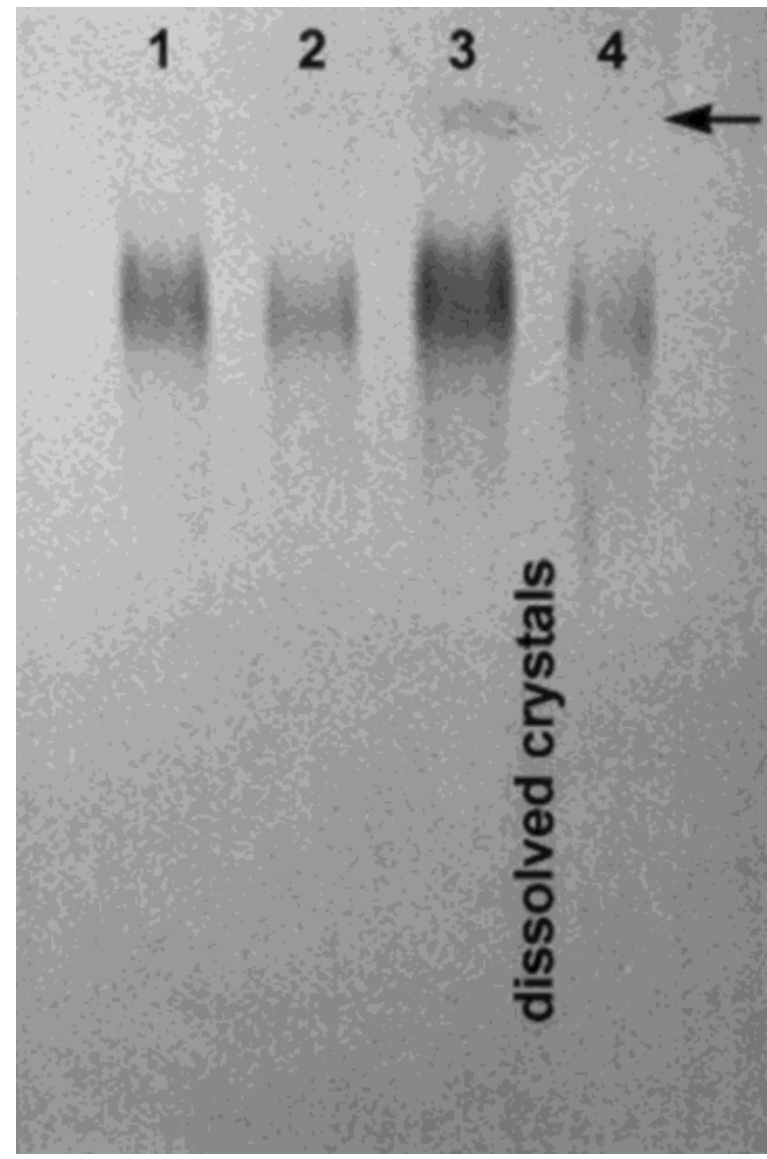

Fig. 1. Agarose gel electrophoresis and immunoblotting (anti apoB) of dissolved LDL crystals (lanes 3 and 4). The control lanes show native LDL solutions in the following dilutions: lane 1: LDL-5, $2 \mu \mathrm{g} / \mathrm{ml}$, lane 2: LDL-2, $0.5 \mu \mathrm{g} / \mathrm{ml}$. The sample application slot is marked by an arrow.

nondichroitic crystals after 1 day, which grew to a size of $80 \times 80 \times 50 \mu \mathrm{m}^{3}$ after 3 weeks.

LDL-2 could be crystallized under the same conditions, yielding rodlike crystals of size $100 \times 100 \times$ $600 \mu^{3}$ that typically had a notch at both ends. The crystals showed strong dichroism and grew to full size after 5 weeks. Both types of crystals were "soft," of the same yellow color as LDL, and floated in the reservoir solution, indicating a low density, as expected for LDL crystals.

\section{Agarose Gel-Electrophoresis}

Theresult of agarosegel electrophoresis and immunoblotting is shown in Figure 1. Dissolved crystals showed a strong signal with anti-human apoB and a mobility similar to LDL solutions (LDL-5 and LDL2). This indicates clearly that the crystals contain apoB. Because no detergents or other delipidating agents were present during preparation and crystallization, the result supports the assumption that the crystals consist of LDL particles.

\section{FTIR Spectroscopy}

To further prove the nature of the crystals, FTIR spectroscopy was used. The result is shown in Figure 2. The absorptions at $1732 \mathrm{~cm}^{-1}$ (a) and $1466 \mathrm{~cm}^{-1}$ (e) correspond to the lipid ester carbonyl stretching mode and the $\mathrm{C}-\mathrm{H}$ torsion, respectively. Both modes aretypical for lipids such as PL, CE , or TG. The band at $1650 \mathrm{~cm}^{-1}$ (b) corresponds to the amide I and the broad absorption in the region of $1510-1560 \mathrm{~cm}^{-1}(\mathrm{~d})$ corresponds to the amide II band. Both arise from the protein backbone.

\section{X-Ray Diffraction}

In contrast to LDL-5, where no diffraction was detectable, we observed reproducible diffraction with crystals of LDL-2 (Fig. 3). The observed diffraction pattern showed well-resolved series of discrete spots and single spots up to $29 \AA$. The spacings within a row corresponded to distances between 382 (Fig. 3c) and $210 \AA$ (Fig. $3 \mathrm{~b}$ ). The spacing of consecutive rows was $\sim 170 \AA$. With the 98 strongest reflections from eight frames, collected in steps of $10^{\circ}\left(\Delta \mathrm{Phi}=0.1^{\circ}\right)$, further evaluation of the data with XDS was attempted. Autoindexing based on the statistics of observed difference vectors resulted in parameters for the reduced cell of $a=171 \AA, b=438 \AA$, $C=519 \AA, \alpha=102^{\circ}, \beta=99^{\circ}$, and $\gamma=91^{\circ}$ if indexed in $P 1$. These results are in general agreement with values expected for native LDL particles, which have a cal culated diameter of $\sim 200 \AA$ (Table 1), assuming a spherical shape.

\section{DISCUSSION}

In this study we report the crystallization and preliminary X-ray diffraction of native LDL partides. To confirm that the crystals really consist of LDL particles, we used three independent techniques: X-ray scattering, FTIR, and gel electrophoresis. In agarose gels, dissolved crystals exhibited the same electrophoretic mobility as native LDL solutions. One single band was observed by immunoblotting with anti-apoB. No signs of oxidative modification was present, in which case the more negatively charged particles would have migrated faster. ${ }^{16} \mathrm{FTIR}$ spectra of the crystals were comparable with spectra of the native LDL solutions, showing typical lipid and protein bands. This indicates clearly that the crystals contained both lipid and protein. These results combined with the unit cell estimation by X-ray diffraction strongly support the view that we have grown crystals of LDL particles.

Moreover, we were able to crystallize two LDL subfractions of different density and particle size. The two subfractions show different crystal forms and grow to different sizes. Up to now, only the crystals of LDL-2 show X-ray diffraction. Whether this is due to the size of the crystals or the fact that crystals of LDL-5 show no dichroism and are possibly disordered remains to be determined. 


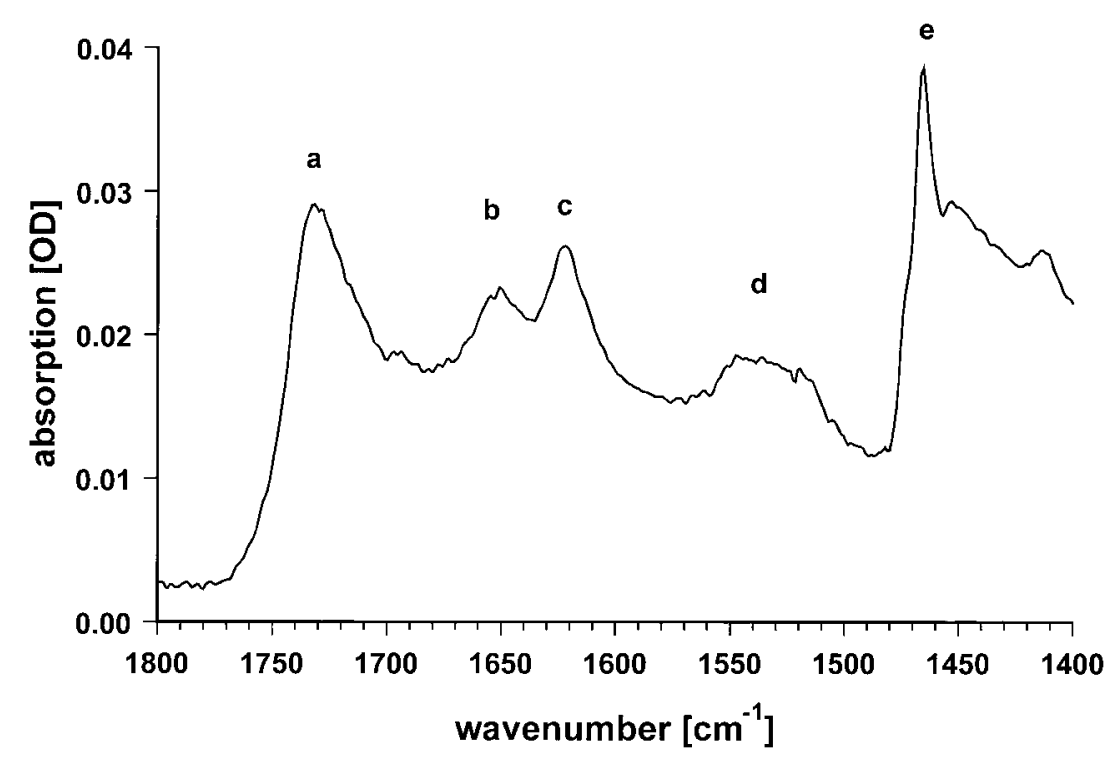

Fig. 2. FTIR spectrum of $\mathrm{LDL}$ crystals dried on $\mathrm{CaF}_{2}$ windows. Bands typical for lipids $(\mathrm{a}, \mathrm{e})$ and protein (b, d) are visible. Band (c) is most probably due to residual buffer substances.

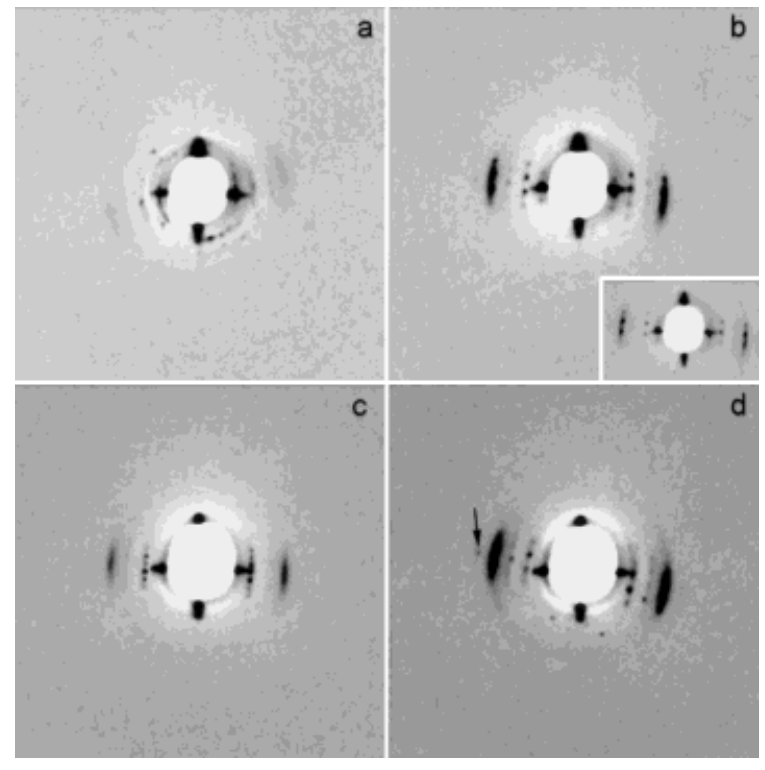

Fig. 3. Synchrotron X-ray diffraction patterns of LDL-2 crystals. The intense arcs visible in (b), (c), and (d) resolve to discrete spots if the image intensity is reduced (inset, b). The arrow in (d) marks the position of the outermost reflection $(29 \AA)$ that could reproducibly be detected. The spacing within a row of reflections corresponds to $382 \AA$ in (c) and $210 \AA$ in (b) and (d). The four intense spots arround the shadow of the beamstop (radius $\sim 80 \AA$ ) are caused by scattering at the collimation slits.

In summary, these data indicate that it should be possible to solve the three-dimensional structure of apoB by X-ray crystallography. This could not be expected a priori because LDL contains a very large amount of different lipids and only $\sim 30 \%$ protein. With the use of subfractions of a well-defined density, however, it proved possible to crystallize particles from both the dense and the buoyant side of the particle spectrum. The main challenge now is to improve the resolution of the crystals by optimizing purification, crystallization, and data collection. Increasing the resolution by establishing cryogenic conditions for data collection could be a feasible next step.

\section{ACKNOWLEDGMENTS}

This work was supported by the Zentrum für klinische Forschung II of the Universitätsklinik Freiburg. We thank S. J otterand for her expert assistance in preparing the LDL subfractions, K. Rahmelow for his help in recording the FTIR spectra, and V. Lamzin from theEMBL Hamburg Outstation at DESY for his support during data collection. We also thank A. Berg and W. Welte for helpful discussions and encouragement.

\section{NOTE ADDED IN PROOF}

Recently a paper by Prassl et al. ${ }^{17}$ came to our attention, reporting the crystallization of an LDL subfraction similar to LDL-2 under quite different conditions.

\section{REFERENCES}

1. Krauss, R.M. Dense low density lipoproteins and coronary artery disease. Am. J . Cardiol. 75:53B-57B, 1995.

2. Baumstark, M.W., Berg, A., Halle, M., Rensing, U.F.E., Roskamm, H., Keul, J. Small dense LDL are a major determinant for the severity of angiographically assessed CAD, even in groups with similar triglyceride levels. Atherosclerosis 109:29-30, 1994.

3. Knott, T.J ., Pease, R.J ., Powell, L.M., Wallis, S.C., Rall, S.C.J ., I nnerarity, T.L., Blackhart, B., Taylor, W.H., Marcel, Y., Milne, R., J ohnson, D., Fuller, M., Losis, A.J ., McCarthy, B.J ., Mahley, R.W., Levy-Wilson, B., Scott, J. Complete 
protein sequence and identification of structural domains of human apolipoprotein B. Nature 323:734-738, 1986.

4. Yang, C.Y., Chen, S.H., Gianturco, S.H., Bradley, W.A., Sparrow, J.T., Tanimura, M., Li, W.H., Sparrow, D.A., DeLoof, H., Rosseneu, M., Lee, F.S., Gu, Z.W., Gotto, A.M., Chan, L. Sequence, structure, receptor-binding domains and internal repeats of human apolipoprotein B-100. Nature 323:738-742, 1986

5. Goormaghtigh, E., De Meutter, J ., Vanloo, B., Brasseur, R., Rosseneu, M., Ruysschaert, J .M. Evaluation of the secondary structure of apo B-100 in low-density lipoprotein (LDL) by infrared spectroscopy. Biochim. Biophys. Acta 1006:147150, 1989.

6. Luzzati, V., Tardieu, A., Aggerbeck, L.P. Structure of serum low-density lipoprotein. I. Solution X-ray scattering study of a hyperlipidemic monkey low-density lipoprotein. J . Mol. Biol. 131:435-473, 1979.

7. Laggner, P., Müller, K.W. The structure of serum lipoproteins as analysed by X-ray small-angle scattering. Q. Rev. Biophys. 11:371-425, 1978.

8. Laggner, P., Kostner, G.M., Degovics, G., Worcester, D.L. Structure of the cholesteryl ester core of human plasma low density lipoproteins: Selective deuteration and neutron small-angle scattering. Proc. Natl. Acad. Sci. USA 81:43894393, 1984.

9. Laggner, P., Kostner, G.M., Rakusch, U., Worcester, D. Neutron small angle scattering on selectively deuterated human plasma low density lipoproteins. J. Biol. Chem. 256:11832-11839, 1981.

10. Baumstark, M.W., Kreutz, W., Berg, A., Frey, I., Keul, J. Structure of human low-density lipoprotein subfractions, determined by X-ray small-angle scattering. Biochim. Biophys. Acta 1037:48-57, 1990.

11. Baumstark, M.W., Kreutz, W., Berg, A., Keul, J . Symmetry of the surface, and structure of the central core of human LDL particles, analyzed by X-ray small angle scattering. In: "Advances in Experimental Medicine and Biology. Vol. 285: Hypercholesterol emia, Hypochol esterolemia, Hypertriglyceridemia, In Vivo Kinetics." Malmendier, C.L., Alaupovic, P., Brewer, B.H.J . (eds.). New York: Plenum Press, 1991:123-130.

12. Lindgren, F.T. Preparative ultracentrifugal laboratory procedures and suggestions for lipoprotein analysis. In: "Analysis of Lipids and Lipoproteins." Perkins, E.G. (ed.). Champain, IL: American Oil Chemists' Society, 1975:204-224.

13. Grafnetter, D., Molinari, E., Lonsky, L. International study on the comparability of Apo A-1 and Apo B methods. Clin. Chim. Acta 189:55-68, 1990.

14. J ancarik, J., Kim, S.H. Sparse matrix sampling: A Screening method for crystallization of proteins. J. Appl. Cryst. 24:409-411, 1991.

15. Kabsch, W. Evaluation of single crystal X-ray diffraction data from a position sensitive detector. J. Appl. Cryst. 21:916-924, 1988

16. Hoff, H.F., O'Neil, J ., Chisolm, G.M. 3d, Cole, T.B., Quehenberger, O., Esterbauer, H., J ürgens, G. Modification of low density lipoprotein with 4-hydroxynonenal induces uptake by macrophages. Arteriosclerosis 9:538-549, 1989.

17. Prassl, R., Chapman, J .M., Nigon, F., Sara, M., Eschenburg, S., Betzel, C., Saxena, A., Laggner, P. Crystallization and preliminary $\mathrm{x}$-ray analysis of a low density lipoprotein from human plasma. J. Biol. Chem. 271:28731-28733, 1966. 